\title{
Aplicação do Método de Avaliação 360 em Residentes Médicos de Ginecologia e Obstetrícia
}

\author{
Application of the $360^{\circ}$ evaluation method in medical residents \\ of Gynecology and Obstetrics
}

Aplicación del método de evaluación $360^{\circ}$ en residentes médicos

de Ginecología y Obstetricia

Carla Alessandra Haber Bastos ${ }^{1 *}$, Nara Macedo Botelho ${ }^{1}$, Márcia Bittar Portella ${ }^{1}$, Camilla Cristina Lisboa do Nascimento ${ }^{1}$.

\section{RESUMO}

Objetivo: Implementar a avaliação $360^{\circ}$ no Programa de Residência Médica em Obstetrícia e Ginecologia de um Hospital de Referência em Obstétrica e Ginecologia, localizado em Belém-PA. Métodos: Pesquisa de caráter exploratório, descritivo, com abordagem quantitativa. Foi desenvolvida nos cenários reais de prática dos médicos residentes e abrangeu todos os setores da tocoginecologia de um hospital de referência em procedimentos obstétricos e ginecológicos, localizado em Belém/PA. Os residentes e preceptores receberam capacitação in loco para participação no estudo. Foi definido como instrumento de avaliação 4 questionários: o Mini Clinical Evaluation Exercise (Mini-CEx), o qual foi utilizado pelos preceptores, além de instrumentos avaliativos do American Board Internal Medicine, que foram utilizados na avaliação pelos pares, pacientes e autoavaliação. Resultados: Foi aplicado um quantitativo total de 92 questionários, sendo 4 tipos diferentes para cada residente, o qual foi possível observar durante uma consulta a sua autoavaliação, a avaliações dos pares, avaliação do preceptor e a avaliação da paciente. Conclusão: Espera-se que esse estudo possa contribuir para futuras possibilidades de ampliação do processo de avaliação dos residentes.

Palavras-chave: Residência Médica, Avaliação, Ginecologia e Obstetrícia.

\begin{abstract}
Objective: To implement the evaluation $360^{\circ}$ in the Medical Residency program in Obstetrics and gynecology of a referral Hospital in obstetrics and gynecology, located in Belém-PA. Methods: Exploratory, descriptive research with quantitative approach. It was developed in the real practice scenarios of resident physicians and covered all sectors of the topology of a referral hospital in obstetric and gynecological procedures, located in Belém/PA. Residents and Preceptors received on-site training for participation in the study. It was defined as an evaluation instrument 4 questionnaires: The Mini Clinical Evaluation Exercise (Mini-CEx), which was used by the preceptors, in addition to evaluative instruments of the American Board Internal Medicine, which were used in the evaluation by Peers, patients and self-assessment. Results: A total quantitative of 92 questionnaires was applied, with 4 different types for each resident, which was possible to observe during a consultation of their self-assessment, peer evaluations, preceptor evaluation and patient evaluation. Conclusion: It is hoped that this study may contribute to future possibilities of expanding the evaluation process of residents.
\end{abstract}

Key words: Medical residency, Evaluation, Obstetrics and Gynecology.

${ }^{1}$ Universidade do Estado do Pará (UEPA), Belém-PA. *E-mail: carlahaber@gmail.com 


\section{RESUMEN}

Objetivo: Implementar la evaluación 360 o en el Programa de Residencia Médica en Obstetricia y Ginecología de un Hospital de Referencia en obstetricia y ginecología, ubicado en Belém-PA. Métodos: Investigación exploratoria y descriptiva con enfoque cuantitativo. Se desarrolló en los escenarios de práctica real de los médicos residentes y cubrió todos los sectores de la topología de un hospital de referencia en procedimientos obstétricos y ginecológicos, ubicado en Belém/PA. Los residentes y preceptores recibieron capacitación in situ para participar en el estudio. Se definió como un instrumento de evaluación 4 cuestionarios: El Mini Ejercicio de Evaluación Clínica (Mini-CEx), que fue utilizado por los preceptores, además de los instrumentos de evaluación de la Junta Americana de Medicina Interna, que se utilizaron en la evaluación por Pares, pacientes y autoevaluación. Resultados: Se aplicó un cuantitativo total de 92 cuestionarios, con 4 tipos diferentes para cada residente, que fue posible observar durante una consulta de su autoevaluación, evaluaciones por pares, evaluación de preceptores y evaluación de pacientes. Conclusión: Se espera que este estudio pueda contribuir a las posibilidades futuras de ampliar el proceso de evaluación de los residentes.

Palabras clave: Residencia médica, Evaluación, Obstetricia y Ginecología.

\section{INTRODUÇÃO}

O Programa de Residência Médica (PRM) constitui uma modalidade de ensino de pós-graduação latu sensu destinada a médicos, caracterizada pelo treinamento em serviço. A residência representa bem mais do que uma busca de aperfeiçoamento da competência profissional, pois, ao ingressar nesta pós-graduação procura-se 0 treinamento em alguma especialidade, internalização de preceitos, normas éticas e 0 desenvolvimento do espírito crítico (SOUSA JCV, 2015). É neste momento que o estudante completa sua formação médica.

Megale LM (2007) destaca que a Residência Médica (RM) baseada em competências já é uma realidade em diversos países do mundo. Visto isso, há a crescente utilização de métodos de avaliação de condutas nesta modalidade de especialização. Quando pontuado o Brasil, também é notória esta preocupação, no qual algumas instituições em diversos estados buscam essa avaliação para além do conhecimento, visto que os mesmos almejam uma formação mais adequada e completa deste médico em formação.

A luz destes conceitos, reflete-se sobre a importância da avaliação $360^{\circ}$, que se destaca como um processo avaliativo de competências largamente difundida pelos livros de gestão de pessoas, sites e revistas especializadas em recursos humanos, sendo considerada uma ferramenta moderna, atual e funcional para avaliar pessoas em diferentes contextos de trabalho e aprendizagem (SILVA JMS, 2016).

Ela é considerada uma das mais adequadas ferramentas de avaliação de competências, incluindo profissionalismo, aprendizado baseado na prática e cuidado com o paciente, podendo ser utilizada para avaliar o conhecimento médico, eliminando, em parte, a subjetividade dos avaliadores (MEGALE LM, 2007).

Tais competências fazem parte de vários currículos de residências mundialmente, e possibilitam a análise do comportamento do médico residente em quaisquer domínios propostos (SILVA JMS, 2016).

Possibilita, além disso, discussões que permitem identificar pontos fortes e fracos e oportunidades de melhorias individuais, a partir, de percepções de vários ângulos: preceptores, pares, pacientes e auto avaliação, e mais do que isso, possibilita a reflexão entre suas próprias percepções e de outras pessoas envolvidas no processo, podendo ter como consequências uma modificação de atitudes e comportamentos dentro e fora das instituições (SILVA JMS, 2016). Ou seja, ela se destaca como sendo um processo avaliativo no qual o avaliado se auto avalia e também recebe feedbacks de todos os que interagem com ele como pacientes, pares e preceptores. Nesse contexto, observa-se a necessidade da identificar como os PRM avaliam o nível de competências adquiridas pelos residentes, com o intuito da melhoria da formação durante o tempo desta especialização. Contudo, é notório que o processo de ensino aprendizagem durante a residência é ainda pouco avaliado e, por conseguinte, estudado (HAUER KE, et al., 2015). 
Reitera-se então, que a prática de aplicação do método de avaliação $360^{\circ}$ no Programa de Residência Médica de Ginecologia e Obstetrícia (PRMGO) almeja à avaliação integral dos médicos residentes, como sendo uma forma de integralizar a avaliação cognitiva isolada (SOUSA JCV, 2015).

Com isto, essa pesquisa apresentou como objetivo implementar a avaliação 360ำ no PRMGO de um Hospital de Referência em Obstétrica e Ginecologia, localizado em Belém-PA.

\section{MÉTODOS}

Pesquisa de caráter exploratório, descritivo, com abordagem quantitativa. O estudo se desenvolveu nos cenários reais de prática dos médicos residentes e abrangeu todos os setores da tocoginecologia de um hospital de referência em procedimentos obstétricos e ginecológicos, localizado em Belém/PA, sendo estes: enfermarias, ambulatórios, centro obstétrico, centro cirúrgico e urgência/emergência (triagem) da ginecologia e obstetrícia. Os residentes e preceptores receberam capacitação in loco para participação no estudo, sendo que os médicos preceptores também foram treinados previamente.

O estudo foi realizado com a população total de médicos residentes de Ginecologia e Obstetrícia e preceptores do Programa de Residência Médica de Ginecologia e Obstetrícia (PRMGO) que estavam devidamente matriculados na Comissão de Residência Médica (COREME) durante a coleta de dados. Além deles, participaram os pacientes assistidos pelo Médico Residente no período de realização da pesquisa, sendo o número determinado pelo número total de médicos residentes. Ressalta-se que não foi coletado o perfil das pacientes assistidas pelos Residentes Médicos (RM) sendo apenas estas identificadas pela sequência alfanumérica, atribuído para o médico que a estava assistindo.

Os critérios de exclusão observados foram os médicos residentes ou preceptores que solicitaram seu desligamento na Comissão da Residência Médica do hospital campo da pesquisa, durante o período do estudo, e os participantes que não tenham assinado o termo de consentimento livre e esclarecido ou não tenha respondido a no mínimo $60 \%$ da ficha utilizada na pesquisa. É importante salientar também que residentes visitantes, oriundos de outros serviços também foram excluídos.

Mediante a autorização do Comitê de Ética e Pesquisa, sob o parecer no 2.766 .099 , foi apresentado o projeto para os preceptores do PRMGO, explicando a metodologia e objetivo da pesquisa, a fim de propiciar maior aproximação entre profissionais e pesquisadora. Foram definidos como instrumentos de avaliação quatro questionários: o Mini Clinical Evaluation Exercise (Mini-CEx), o qual foi utilizado pelos preceptores, além de instrumentos avaliativos do American Board Internal Medicine, que foram utilizados na avaliação pelos pares, pacientes e autoavaliação (NORCINI JJ et al, 2003).

Ressalta-se, além disso, que os instrumentos de avaliação foram utilizados na íntegra com fins de garantir a integridade dos direitos autorais e que cada residente foi avaliado pelo preceptor, pelos pares (ou seja, por outro médico residente), e por um paciente, todos escolhidos de forma aleatória. A coleta de dados foi realizada em duas etapas: treinamento dos preceptores para utilização do instrumento de avaliação $360^{\circ} \mathrm{e}$ Aplicação da avaliação nos residentes.

\section{1) O treinamento dos preceptores para utilização do instrumento de avaliação $360^{\circ}$}

Nesta etapa, a totalidade dos preceptores tiveram o contato com o método e receberam o treinamento de como utilizar a avaliação $360^{\circ}$, antes do início das avaliações. Foi ministrada uma breve aula expositiva de como funciona o método e depois foi apresentado um vídeo explicando o método de avaliação $360^{\circ}$.

\section{2) Aplicação da avaliação nos residentes}

Os médicos residentes foram submetidos à avaliação pelo preceptor, auto avaliação, avaliação pelos pares e avaliação pelos pacientes no mesmo momento, no período de agosto de 2018 e abril de 2019, em ambientes de atendimento diversos.

Além disso, também foi fornecida uma devolutiva sistemática da avaliação aos residentes, proporcionando assim a possibilidade de elaboração de planos de recuperação para a fragilidade identificada. 
Ressalta-se que os residentes foram informados que a avaliação $360^{\circ}$ não foi considerada como avaliação oficial do módulo.

Para análise de dados, foi criado um banco em planilha no software Excel/Office 2010, o qual foi exportado para o programa GraphPad prism 6, com todas as variáveis descritas nos quatros questionários estruturados, separadas por ano de residência médica cursado, que serão submetidos à análise estatística. Esta pesquisa recebeu autorização para ser desenvolvida pelo Comitê de Ética em Pesquisa da Fundação Santa Casa de Misericórdia do Pará, sob o parecer nํ⒉766.099.

\section{RESULTADOS E DISCUSSÃO}

Foi aplicado um quantitativo total de 60 questionários, sendo quatro tipos diferentes para cada residente, o qual foi possível observar durante uma consulta a sua autoavaliação, a avaliações dos pares, avaliação do preceptor e a avaliação da paciente.

Participaram da pesquisa um quantitativo total de 15 residentes, sendo $40 \%$ (seis) do primeiro ano, $40 \%$ (seis) do segundo e $20 \%$ (três) do terceiro. Do total, a maioria composta por mulheres (60\%).

Além deles, participaram 12 médicos participantes do PRMGO, todos em situação regular pelo Conselho Regional de Medicina do estado do Pará, sendo 69,23\% do sexo feminino e média de 14 anos de profissão $( \pm 7,597)$.

Ressalta-se que 3 preceptores ficaram responsáveis pela avaliação de 2 residentes e 9 preceptores pela avaliação de 01 residente somente, representando então a totalidades dos RM avaliados.

Destaca-se que a profissão médica apresenta a tendência de à feminização, visto que, em 2016, 54,9\% dos registros médicos foram de mulheres, enquanto, em 2000, foram 44\% (SCHEFFER M, et al., 2018).

Todos os questionários foram respondidos em sua íntegra, por meio de escores pontuados na escala de Likert, de 1 a 10 na análise quantitativa, sendo 1 a menor nota e 10 a maior nota.

As avaliações ocorreram no mês de agosto de 2018 a abril de 2019 e tiveram como cenários de avaliação as Enfermarias (53,33\%), Centro Obstétrico $(26,66 \%)$ e Triagem Obstétrica $(20 \%)$ do hospital participante da pesquisa.

Sendo que 14 (93,33\%) dos casos avaliados tratava-se de primeira consulta dentro do cenário e a mesma foi executada pelo residente avaliado, no qual foi realizada Anamnese e Exame Físico Completo.

\section{Avaliação dos preceptores}

A primeira etapa foi caracterizada pelo treinamento do corpo de preceptoria do PRMGO para a aplicação das avalições $360^{\circ}$.

Pode-se constatar que $100 \%$ dos preceptores não tinham conhecimento desse processo, como realizar a aplicação e a importância do mesmo para o processo de formação. Em aspecto geral, os preceptores avaliaram, na maioria dos itens avaliados, os residentes dentro das expectativas.

É perceptível que os R1 estiveram acima das expectativas nos aspectos "humanismo", "explicações e orientações", "respeito", "trabalho em equipe", "pontualidade" e "assiduidade", já os R2 e R3 apresentaram a média menor nos aspectos "raciocínio clínico" e "trabalho em equipe", respectivamente.

Resultados dos estudos de Fabricio TNBD (2018) demostram que que no mesmo tipo de avaliação implantada na RM de ginecologia e obstetrícia em um hospital de Natal/RN, os escores médios obtidos por residentes do primeiro ano, foram menores quando comparados aos obtidos pelos R2 e R3 nos na maioria dos aspectos analisados.

A Tabela 1 apresenta as médias obtidas na aplicação da mini-avaliação clínica (MINI-CEX), realizada pelos preceptores da residência médica de ginecologia e obstetrícia para cada item avaliado, para os residentes nos três anos de residência. 
Tabela 1 - Média e Desvio Padrão de cada ano da RM e total dos escores obtidos na escala Likert na avaliação dos preceptores dentro de todos os aspectos avaliados. Belém-PA, 2019.

\begin{tabular}{|c|c|c|c|c|}
\hline ASPECTO & R1 & $\mathbf{R 2}$ & R3 & TOTAL \\
\hline ANAMNESE & $\begin{array}{c}7,83 \\
( \pm 1,471)\end{array}$ & $\begin{array}{c}7,33 \\
( \pm 2,160)\end{array}$ & $\begin{array}{c}7,33 \\
( \pm 4,795)\end{array}$ & $\begin{array}{c}7,15 \\
( \pm 2,378)\end{array}$ \\
\hline EXAME CLÍNICO & $\begin{array}{c}7,16 \\
( \pm 1,169)\end{array}$ & $\begin{array}{c}7,66 \\
( \pm 2,503)\end{array}$ & $\begin{array}{c}7,33 \\
( \pm 4,795)\end{array}$ & $\begin{array}{c}7,10 \\
( \pm 2,523)\end{array}$ \\
\hline HABILIDADES CLÍNICAS & $\begin{array}{c}7,50 \\
( \pm 1,516)\end{array}$ & $\begin{array}{c}7,00 \\
( \pm 2,280)\end{array}$ & $\begin{array}{c}7,33 \\
( \pm 4,795)\end{array}$ & $\begin{array}{c}6,95 \\
( \pm 2,432)\end{array}$ \\
\hline RACIOCÍNIO CLÍNICO & $\begin{array}{c}7,50 \\
( \pm 1,643)\end{array}$ & $\begin{array}{c}6,83 \\
( \pm 2,562)\end{array}$ & $\begin{array}{c}7,33 \\
( \pm 4,795)\end{array}$ & $\begin{array}{c}7,00 \\
( \pm 2,487)\end{array}$ \\
\hline HUMANISMO & $\begin{array}{c}8,83 \\
( \pm 1,329)\end{array}$ & $\begin{array}{c}8,00 \\
( \pm 3,464)\end{array}$ & $\begin{array}{c}7,33 \\
( \pm 4,795)\end{array}$ & $\begin{array}{c}7,82 \\
( \pm 3,136)\end{array}$ \\
\hline EXPLICAÇÕES E ORIENTAÇÕES & $\begin{array}{c}8,50 \\
( \pm 1,378)\end{array}$ & $\begin{array}{c}6,50 \\
( \pm 3,209)\end{array}$ & $\begin{array}{c}7,00 \\
( \pm 4,573)\end{array}$ & $\begin{array}{c}7,03 \\
( \pm 2,966)\end{array}$ \\
\hline ORGANIZAÇÃO E EFICIÊNCIA & $\begin{array}{c}7,83 \\
( \pm 2,136)\end{array}$ & $\begin{array}{c}7,33 \\
( \pm 3,265)\end{array}$ & $\begin{array}{c}7,33 \\
( \pm 4,795)\end{array}$ & $\begin{array}{c}7,18 \\
( \pm 2,957)\end{array}$ \\
\hline RESPEITO & $\begin{array}{c}9,33 \\
( \pm 1,211)\end{array}$ & $\begin{array}{c}8,00 \\
( \pm 3,521)\end{array}$ & $\begin{array}{c}6,66 \\
( \pm 5,228)\end{array}$ & $\begin{array}{c}7,84 \\
( \pm 3,375)\end{array}$ \\
\hline TRABALHO EM EQUIPE & $\begin{array}{c}9,33 \\
( \pm 0,816)\end{array}$ & $\begin{array}{c}7,83 \\
( \pm 3,544)\end{array}$ & $\begin{array}{c}4,66 \\
( \pm 3,511)\end{array}$ & $\begin{array}{c}7,59 \\
( \pm 3,400)\end{array}$ \\
\hline PONTUALIDADE & $\begin{array}{c}8,16 \\
( \pm 1,834)\end{array}$ & $\begin{array}{c}8,50 \\
( \pm 2,073)\end{array}$ & $\begin{array}{c}8,33 \\
( \pm 4,500)\end{array}$ & $\begin{array}{c}8,00 \\
( \pm 2,247)\end{array}$ \\
\hline ASSIDUIDADE & $\begin{array}{c}9,50 \\
( \pm 0,547)\end{array}$ & $\begin{array}{c}9,00 \\
( \pm 2,000)\end{array}$ & $\begin{array}{c}8,33 \\
( \pm 4,500)\end{array}$ & $\begin{array}{c}8,63 \\
( \pm 2,398)\end{array}$ \\
\hline
\end{tabular}

${ }^{*}$ Legenda de cor: verde-acima da expectativa; amarelo-atende as expectativas; vermelho-abaixo das expectativas.

Fonte: Bastos CAH, Botelho NM, et al., 2019.

\section{Autoavaliação e avaliação pelos pares}

Observa-se que, independente do ano e do cenário em que ocorreu a avaliação, a maioria do quantitativo (66,6\%) apresentaram insegurança quando realizada a avaliação pessoal sobre os seus conhecimentos médicos, sendo uma média total dessa temática de 7,24 pontos na escala Likert. 
Um estudo de Fabricio TNBD (2018) mostra na análise da autoavaliação, que existe uma tendência entre os residentes dos anos iniciais a se avaliarem com escores mais altos, enquanto os residentes dos anos finais, se auto avaliam com escores mais baixos, como também analisado neste estudo, descrito na tabela anterior.

Como apresentado na Tabela 2, os escores médios obtidos por residentes do primeiro ano, foram menores quando comparados aos do segundo e do terceiro nos seguintes itens: "conhecimento médico" e "manejo de problemas complexos".

Tabela 2 - Média e Desvio Padrão dos escores obtidos na escala Likert na autoavaliação dos aspectos avaliados, por ano de residência. Belém-PA, 2019.

\begin{tabular}{|c|c|c|c|c|}
\hline ASPECTO & R1 & R2 & R3 & TOTAL \\
\hline RESPEITO & $\begin{array}{c}10,00 \\
( \pm 0,000)\end{array}$ & $\begin{array}{c}9,83 \\
( \pm 0,408)\end{array}$ & $\begin{array}{c}10,00 \\
( \pm 0,000)\end{array}$ & $\begin{array}{c}9,45 \\
( \pm 2,132)\end{array}$ \\
\hline CONHECIMENTO MÉDICO & $\begin{array}{c}6,83 \\
( \pm 1,471)\end{array}$ & $\begin{array}{c}7,83 \\
( \pm 1,834)\end{array}$ & $\begin{array}{c}7,66 \\
( \pm 3,947)\end{array}$ & $\begin{array}{c}7,24 \\
( \pm 2,027)\end{array}$ \\
\hline $\begin{array}{l}\text { MANEJO NO ATENDIMENTO AO } \\
\text { PACIENTE }\end{array}$ & $\begin{array}{c}8,58 \\
( \pm 1,114)\end{array}$ & $\begin{array}{c}8,00 \\
( \pm 1,788)\end{array}$ & $\begin{array}{c}9,33 \\
( \pm 4,760)\end{array}$ & $\begin{array}{c}8,09 \\
( \pm 2,124)\end{array}$ \\
\hline INTEGRIDADE & $\begin{array}{c}9,50 \\
( \pm 0,836)\end{array}$ & $\begin{array}{c}9,50 \\
( \pm 0,836)\end{array}$ & $\begin{array}{c}10,00 \\
( \pm 0,000)\end{array}$ & $\begin{array}{c}9,15 \\
( \pm 1,992)\end{array}$ \\
\hline $\begin{array}{l}\text { MANEJO DE PROBLEMAS } \\
\text { COMPLEXOS }\end{array}$ & $\begin{array}{c}7,50 \\
( \pm 1,378)\end{array}$ & $\begin{array}{c}7,66 \\
( \pm 1,505)\end{array}$ & $\begin{array}{c}9,00 \\
( \pm 1,000)\end{array}$ & $\begin{array}{c}7,53 \\
( \pm 1,932)\end{array}$ \\
\hline COMPAIXÃO & $\begin{array}{c}9,96 \\
( \pm 0,081)\end{array}$ & $\begin{array}{c}9,66 \\
( \pm 0,516)\end{array}$ & $\begin{array}{c}8,66 \\
( \pm 2,309)\end{array}$ & $\begin{array}{c}9,22 \\
( \pm 2,225)\end{array}$ \\
\hline RESPONSABILIDADE & $\begin{array}{c}10,00 \\
( \pm 0,000)\end{array}$ & $\begin{array}{c}9,66 \\
( \pm 0,516)\end{array}$ & $\begin{array}{c}8,66 \\
( \pm 1,527)\end{array}$ & $\begin{array}{c}9,22 \\
( \pm 2,180)\end{array}$ \\
\hline RESOLUÇÃO DE PROBLEMAS & $\begin{array}{c}9,15 \\
( \pm 0,966)\end{array}$ & $\begin{array}{c}8,16 \\
( \pm 1,722)\end{array}$ & $\begin{array}{c}8,00 \\
( \pm 2,000)\end{array}$ & $\begin{array}{c}8,13 \\
( \pm 2,192)\end{array}$ \\
\hline HABILIDADES & $\begin{array}{c}8,98 \\
( \pm 0,872)\end{array}$ & $\begin{array}{c}8,16 \\
( \pm 1,722)\end{array}$ & $\begin{array}{c}9,00 \\
( \pm 1,000)\end{array}$ & $\begin{array}{c}8,21 \\
( \pm 2,130)\end{array}$ \\
\hline COMPETÊNCIAS GERAIS & $\begin{array}{c}9,15 \\
( \pm 0,966)\end{array}$ & $\begin{array}{c}8,33 \\
( \pm 1,366)\end{array}$ & $\begin{array}{c}7,66 \\
( \pm 2,081)\end{array}$ & $\begin{array}{c}8,18 \\
( \pm 2,089)\end{array}$ \\
\hline $\begin{array}{c}\text { RELACIONAMENTO COM A EQUIPE } \\
\text { DE SAÚDE }\end{array}$ & $\begin{array}{c}10,00 \\
( \pm 0,000)\end{array}$ & $\begin{array}{c}9,33 \\
( \pm 0,516)\end{array}$ & $\begin{array}{c}10,00 \\
( \pm 0,000)\end{array}$ & $\begin{array}{c}9,22 \\
( \pm 2,114)\end{array}$ \\
\hline PONTUALIDADE & $\begin{array}{c}9,13 \\
( \pm 0,952)\end{array}$ & $\begin{array}{c}9,33 \\
( \pm 0,516)\end{array}$ & $\begin{array}{c}7,33 \\
( \pm 1,547)\end{array}$ & $\begin{array}{c}8,63 \\
( \pm 1,969)\end{array}$ \\
\hline ASSIDUIDADE & $\begin{array}{c}9,63 \\
( \pm 0,496)\end{array}$ & $\begin{array}{c}9,50 \\
( \pm 0,547)\end{array}$ & $\begin{array}{c}9,66 \\
( \pm 0,577)\end{array}$ & $\begin{array}{c}9,13 \\
( \pm 1,987)\end{array}$ \\
\hline
\end{tabular}

${ }^{*}$ Legenda de cor: verde-acima da expectativa; amarelo-atende as expectativas; vermelho-abaixo das expectativas.

Fonte: Bastos CAH, Botelho NM, et al., 2019. 
Entretanto, como explanado no Gráfico 1, a média dos escores para a mesma escala quando comparado a avaliação individual com a avaliação dos seus pares, dentro da mesma temática, se apresentou de forma diferenciada podendo destacar os aspectos "conhecimento médico", "manejo de problemas complexos" e "competências gerais".

Gráfico 1 - Média geral dos escores obtidos na escala Likert para autovaliação e avaliação pelos pares. Belém-PA, 2019.

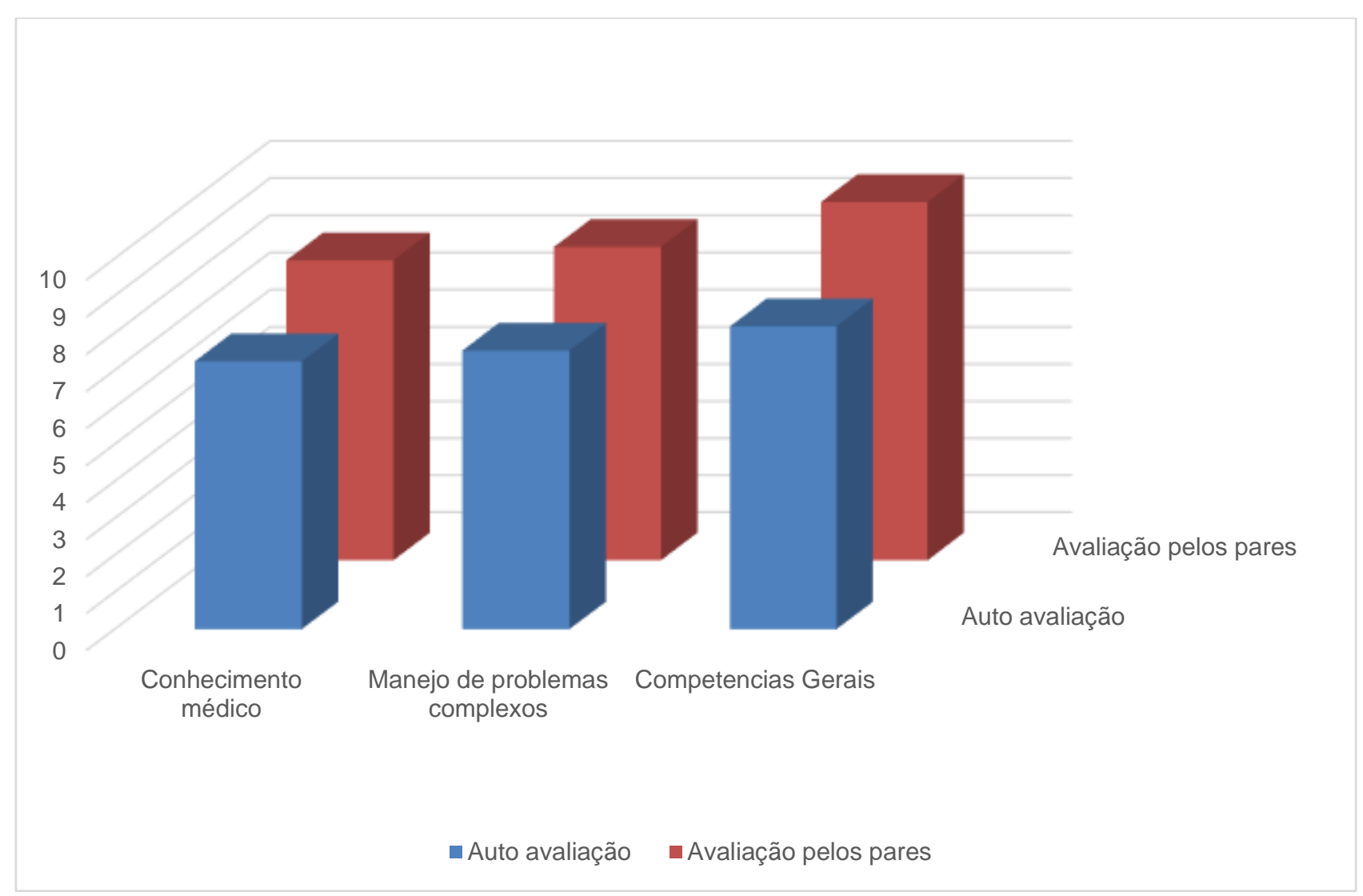

Fonte: Bastos CAH, Botelho NM, et al., 2019.

Abreu-Reis $P$, et al. (2019) reflete em suas pesquisas que o residente médico está acometido a uma série de cobranças por parte de seus preceptores, da sociedade, das múltiplas funções exercidas juntamente com as extensas jornadas de trabalho, tarefas obrigatórias e maior responsabilidade como profissional, com altas demandas por competência e eficiência.

Isto é, é notória a percepção da insegurança por parte dos mesmos para a realização da sua autoavaliação, todavia conseguem avaliar de forma positiva os seus colegas de residência em todos os aspectos analisados (Tabela 3). Em vista disso, é destacada a percepção que os médicos apresentam tal insegurança não pelo desconhecimento de saberes clínicos, mas sim pela ansiedade de futuras cobranças atreladas a prática da conduta médica de forma inadequada.

Ressalta-se ainda, que se pode atrelar esta insegurança com as pesadas demandas físicas e emocionais do curso, a supressão emocional, e, com isso, um possível distanciamento com os pacientes por não se sentirem totalmente seguros de suas práticas (TORRES AR, et al., 2019).

Em vista da tabela anterior, observa-se que apenas o aspecto "pontualidade" (escore total 7,72) se encontrou na escala que atende a expectativa, quando analisada a média total dos escores. Os demais apresentaram-se acima da expectativa. 
Tabela 3 - Média Geral e Desvio Padrão Geral dos escores obtidos na escala Likert na avaliação dos pares dentro de todos os aspectos avaliados nos níveis de expectativa. Belém-PA, 2019.

\begin{tabular}{|c|c|c|c|c|}
\hline ASPECTO & $\mathbf{R 1}$ & $\mathbf{R 2}$ & R3 & TOTAL \\
\hline RESPEITO & $\begin{array}{c}9,16 \\
( \pm 0,752)\end{array}$ & $\begin{array}{c}9,00 \\
( \pm 0,894)\end{array}$ & $\begin{array}{c}6,33 \\
( \pm 3,685)\end{array}$ & $\begin{array}{c}8,26 \\
( \pm 2,163)\end{array}$ \\
\hline CONHECIMENTO MÉDICO & $\begin{array}{c}8,66 \\
( \pm 0,516)\end{array}$ & $\begin{array}{c}8,66 \\
( \pm 1,032)\end{array}$ & $\begin{array}{c}7,33 \\
( \pm 4,203)\end{array}$ & $\begin{array}{c}8,09 \\
( \pm 2,061)\end{array}$ \\
\hline $\begin{array}{c}\text { MANEJO NO ATENDIMENTO AO } \\
\text { PACIENTE }\end{array}$ & $\begin{array}{c}9,16 \\
( \pm 0,752)\end{array}$ & $\begin{array}{c}8,83 \\
( \pm 1,147)\end{array}$ & $\begin{array}{c}8,33 \\
( \pm 4,349)\end{array}$ & $\begin{array}{c}8,45 \\
( \pm 2,086)\end{array}$ \\
\hline INTEGRIDADE & $\begin{array}{c}9,16 \\
( \pm 1,169)\end{array}$ & $\begin{array}{c}8,83 \\
( \pm 1,169)\end{array}$ & $\begin{array}{c}8,33 \\
( \pm 4,349)\end{array}$ & $\begin{array}{c}8,46 \\
( \pm 1,965)\end{array}$ \\
\hline $\begin{array}{l}\text { MANEJO DE PROBLEMAS } \\
\text { COMPLEXOS }\end{array}$ & $\begin{array}{c}8,66 \\
( \pm 0,516)\end{array}$ & $\begin{array}{c}9,16 \\
( \pm 0,752)\end{array}$ & $\begin{array}{c}8,66 \\
( \pm 4,358)\end{array}$ & $\begin{array}{c}8,55 \\
( \pm 1,911)\end{array}$ \\
\hline COMPAIXÃO & $\begin{array}{c}9,50 \\
( \pm 0,836)\end{array}$ & $\begin{array}{c}9,50 \\
( \pm 0,836)\end{array}$ & $\begin{array}{c}7,33 \\
( \pm 4,203)\end{array}$ & $\begin{array}{c}8,78 \\
( \pm 2,191)\end{array}$ \\
\hline RESPONSABILIDADE & $\begin{array}{c}9,66 \\
( \pm 0,516)\end{array}$ & $\begin{array}{c}9,16 \\
( \pm 1,329)\end{array}$ & $\begin{array}{c}6,33 \\
( \pm 3,685)\end{array}$ & $\begin{array}{c}8,50 \\
( \pm 2,391)\end{array}$ \\
\hline RESOLUÇÃO DE PROBLEMAS & $\begin{array}{c}8,66 \\
( \pm 0,516)\end{array}$ & $\begin{array}{c}9,16 \\
( \pm 0,983)\end{array}$ & $\begin{array}{c}7,66 \\
( \pm 4,203)\end{array}$ & $\begin{array}{c}8,37 \\
( \pm 2,074)\end{array}$ \\
\hline HABILIDADES & $\begin{array}{c}8,66 \\
( \pm 0,516)\end{array}$ & $\begin{array}{c}8,83 \\
( \pm 1,169)\end{array}$ & $\begin{array}{c}8,00 \\
( \pm 3,685)\end{array}$ & $\begin{array}{c}8,28 \\
( \pm 2,112)\end{array}$ \\
\hline COMPETÊNCIAS GERAIS & $\begin{array}{c}9,00 \\
( \pm 0,632)\end{array}$ & $\begin{array}{c}9,16 \\
( \pm 0,983)\end{array}$ & $\begin{array}{c}7,66 \\
( \pm 4,272)\end{array}$ & $\begin{array}{c}8,48 \\
( \pm 2,106)\end{array}$ \\
\hline $\begin{array}{c}\text { RELACIONAMENTO COM A EQUIPE } \\
\text { DE SAÚDE }\end{array}$ & $\begin{array}{c}8,83 \\
( \pm 1,169)\end{array}$ & $\begin{array}{c}9,50 \\
( \pm 0,547)\end{array}$ & $\begin{array}{c}7,66 \\
( \pm 4,193)\end{array}$ & $\begin{array}{c}8,63 \\
( \pm 2,005)\end{array}$ \\
\hline PONTUALIDADE & $\begin{array}{c}8,50 \\
( \pm 1,378)\end{array}$ & $\begin{array}{c}9,16 \\
( \pm 0,983)\end{array}$ & $\begin{array}{c}3,00 \\
( \pm 2,262)\end{array}$ & $\begin{array}{c}7,72 \\
( \pm 2,797)\end{array}$ \\
\hline ASSIDUIDADE & $\begin{array}{c}8,83 \\
( \pm 1,169)\end{array}$ & $\begin{array}{c}9,66 \\
( \pm 0,816)\end{array}$ & $\begin{array}{c}3,66 \\
( \pm 3,593)\end{array}$ & $\begin{array}{c}8,18 \\
( \pm 2,916)\end{array}$ \\
\hline
\end{tabular}

*Legenda de cor: verde-acima da expectativa; amarelo-atende as expectativas; vermelho-abaixo das expectativas.

Fonte: Bastos CAH, Botelho NM, et al., 2019.

\section{Avaliação dos pacientes}

Quando os residentes foram avaliados pelas suas respectivas pacientes, é perceptível que os médicos apresentam uma significativa diminuição nos aspectos gerais em atendimento clínico dentro com o passar dos anos dentro da RM, como apresentado na Tabela 5. 
Tabela 5 - Média de cada ano da RM e Geral e Desvio Padrão de cada ano da RM e Total dos escores obtidos na escala Likert na avaliação dos pacientes dentro de todos os aspectos avaliados. Belém, 2019.

\begin{tabular}{|c|c|c|c|c|}
\hline ASPECTO & R1 & $\mathbf{R 2}$ & R3 & TOTAL \\
\hline $\begin{array}{c}\text { FALA TUDO PARA VOCÊ SOBRE } \\
\text { SUA DOENÇA? }\end{array}$ & $\begin{array}{c}9,50 \\
( \pm 0,836)\end{array}$ & $\begin{array}{c}6,50 \\
( \pm 3,563)\end{array}$ & $\begin{array}{c}7,33 \\
( \pm 4,203)\end{array}$ & $\begin{array}{c}7,15 \\
( \pm 3,227)\end{array}$ \\
\hline CUMPRIMENTA CALOROSAMENTE? & $\begin{array}{c}9,00 \\
( \pm 2,000)\end{array}$ & $\begin{array}{c}7,00 \\
( \pm 3,521)\end{array}$ & $\begin{array}{c}7,33 \\
( \pm 4,203)\end{array}$ & $\begin{array}{c}7,54 \\
( \pm 3,081)\end{array}$ \\
\hline TRATA VOCÊ NO MESMO NÍVEL? & $\begin{array}{c}9,50 \\
( \pm 1,224)\end{array}$ & $\begin{array}{c}7,83 \\
( \pm 3,060)\end{array}$ & $\begin{array}{c}9,33 \\
( \pm 4,760)\end{array}$ & $\begin{array}{c}8,48 \\
( \pm 2,590)\end{array}$ \\
\hline $\begin{array}{c}\text { DEIXA VOCÊ CONTAR SUA } \\
\text { HISTÓRIA? }\end{array}$ & $\begin{array}{c}9,16 \\
( \pm 2,041)\end{array}$ & $\begin{array}{c}7,16 \\
( \pm 3,816)\end{array}$ & $\begin{array}{c}6,00 \\
( \pm 4,795)\end{array}$ & $\begin{array}{c}7,55 \\
( \pm 3,474)\end{array}$ \\
\hline $\begin{array}{l}\text { MOSTRA INTERESSE EM VOCÊ } \\
\text { COMO PESSOA? }\end{array}$ & $\begin{array}{c}5,50 \\
( \pm 3,728)\end{array}$ & $\begin{array}{c}5,00 \\
( \pm 3,633)\end{array}$ & $\begin{array}{c}4,66 \\
( \pm 3,696)\end{array}$ & $\begin{array}{c}5,10 \\
( \pm 3,299)\end{array}$ \\
\hline $\begin{array}{l}\text { TEM O CUIDADO DE FALAR O QUE } \\
\text { VAI FAZER QUANDO ESTÁ TE } \\
\text { EXAMINANDO E O QUE } \\
\text { ENCONTROU APÓS EXAMINAR? }\end{array}$ & $\begin{array}{c}9,50 \\
( \pm 0,836)\end{array}$ & $\begin{array}{c}6,50 \\
( \pm 4,037)\end{array}$ & $\begin{array}{c}7,66 \\
( \pm 4,193)\end{array}$ & $\begin{array}{c}7,24 \\
( \pm 3,491)\end{array}$ \\
\hline $\begin{array}{l}\text { CONVERSA SOBRE } \\
\text { POSSIBILIDADES DE } \\
\text { TRATAMENTO? }\end{array}$ & $\begin{array}{c}8,33 \\
( \pm 2,875)\end{array}$ & $\begin{array}{c}5,66 \\
( \pm 3,669)\end{array}$ & $\begin{array}{c}3,33 \\
( \pm 2,380)\end{array}$ & $\begin{array}{c}6,10 \\
( \pm 3,477)\end{array}$ \\
\hline $\begin{array}{l}\text { ESTIMULA VOCÊ A FAZER } \\
\text { PERGUNTAS? }\end{array}$ & $\begin{array}{c}7,66 \\
( \pm 3,011)\end{array}$ & $\begin{array}{c}5,50 \\
( \pm 3,728)\end{array}$ & $\begin{array}{c}7,00 \\
( \pm 4,112)\end{array}$ & $\begin{array}{c}6,43 \\
( \pm 3,180)\end{array}$ \\
\hline $\begin{array}{l}\text { MOSTRA-SE DISPONÍVEL PARA } \\
\text { NOVAS EXPLICAÇÕES OU AJUDAR } \\
\text { EM OUTROS MOMENTOS? }\end{array}$ & $\begin{array}{c}7,00 \\
( \pm 3,464)\end{array}$ & $\begin{array}{c}5,33 \\
( \pm 3,983)\end{array}$ & $\begin{array}{c}6,66 \\
( \pm 4,163)\end{array}$ & $\begin{array}{c}6,11 \\
( \pm 3,338)\end{array}$ \\
\hline
\end{tabular}

Fonte: Bastos CAH, Botelho NM, et al., 2019.

Ainda analisando a tabela supracitada, observa-se que os pacientes apresentam-se, em geral, abaixo da expectativa (média de 5,10), quanto ao médico apresentar empatia ao caso durante 0 atendimento, demonstrado com o questionamento "mostra interesse em você como pessoa?", sendo destacados pelas médias na escala Likert 5,50 para os R1, 5 para os R2 e 4,66 para os R3.

Nascimento HCF, et al. (2018) reflete que a empatia se apresenta como elo fundamental para a relação médico-paciente. Isto é, ela não é significativa apenas na percepção do que a enfermidade significa para o paciente, mas também em como o médico consegue transmitir a compreensão integral da situação. Com isso, entende-se que a empatia visa também promover um feedback da satisfação do paciente para o médico.

Torres AR, et al. (2019) relata em seus estudos a importância da prática dos preceptores médicos em ajudar os residentes em manter ou aumentar a empatia pelos pacientes, ao lado de outros valores humanitários.

Além disso, a empatia é vista como um dos principais objetivos dentro do ensino médico, visto que esta está atrelada a uma melhor acurácia diagnóstica, a adesão e a resposta ao tratamento, assim como a satisfação do paciente e do médico em todo o processo (SHAPIRO J, 2008).

Entretanto, os pacientes avaliaram de forma geral com escores acima das expectativas, outros aspectos, tendo destaque para os questionamentos "trata você no mesmo nível?" e "deixa você contar a sua história?", com médias gerais respectivas de 8,48 e 7,55.

Em vista disso, reflete-se a necessidade de ampliar práticas pedagógicas de forma inovadora, que apresentem a integralidade do ser humano dentro das disciplinas com o intuito de motivar o residente a se tornar um profissional mais reflexivo, engajado, sensível e cuidadoso de forma integral com o paciente e seus familiares. 


\section{Boas práticas na residência médica: contribuições da avaliação no ensino e formações de profissionais}

A prática de inserção de qualquer metodologia pedagógica torna-se desafiadora na área da saúde para qualquer meio que apresente uma alta demanda de atendimento.

Quando destacada a avaliação $360^{\circ}$, o maior desafio observado foi a necessidade de uma capacitação permanente de todos os participantes para a aplicação em maior qualidade da mesma.

Tal avaliação é apresentada como instrumento dinâmico que pode ser aplicado preceptor durante o atendimento rotineiro, não interferindo na rotina médica do serviço e principalmente não usando o paciente como objeto de estudo. Isto é, o objeto de avaliação é identificar e corrigir deficiências de desempenho do residente.

Ressalta-se, com isso, a necessidade de o preceptor desenvolver estratégias que otimizem o processo formativo, integrando valores e atitudes pautados na cidadania, na ideia de controle social capazes de influenciar a trajetória desses futuros profissionais (SOUZA SV e FERREIRA BJ, 2019).

Quando destacada o hospital no qual foi realizado o estudo, por trata-ser de um hospital de ensino e de referência obstétrica no estado do Pará, a implementação do método foi destacada como forma inovadora para identificar de pontos fortes e frágeis na formação dos residentes, podendo fazer o exercício de reflexão por parte dos preceptores e da coordenação de residentes para as possíveis resoluções e, consequentemente, a melhoria da formação dos mesmos.

\section{CONSIDERAÇÕES FINAIS}

Espera-se que esse estudo possa contribuir para futuras possibilidades de ampliação do processo de avaliação dos residentes, visto que, a partir desse, foi possível observar as condutas de atendimentos dos médicos residentes. Com a pesquisa, foi possível identificar a necessidade de intensificar o lado humanístico dos mesmos, para maximizar a melhoria de condutas dos residentes médicos.

\section{REFERÊNCIAS}

1. ABREU-REIS P, et al. Aspectos psicológicos e qualidade de vida na Residência Médica. Revista de Colégio Brasileiro de Cirurgiões, 2019; 46(1): 1-6.

2. FABRICIO TNBD. Experiência de implantação da avaliação 360 graus e feedback entre residentes de ginecologia e obstetrícia. Dissertação (Mestrado Profissional em Ensino na Saúde) - Curso de Medicina. Universidade Federal do Rio Grande do Norte, Natal, 2018; 68p.

3. HAUER KE, et al. Reviewing resident's competence: a qualitative study of the role of clinical competence committees in performance assessment. Academic medicine, 2015; 90 (8): 1084-1092.

4. MEGALE LM. Pediatria: proposta de instrumento de avaliação discente no internato. Dissertação (Mestrado Profissional em Ciências da Saúde) - Curso de Medicina. Universidade Federal de Minas Gerais, Belo Horizonte, 2007; 98 p.

5. NASCIMENTO HCF, et al. Análise dos níveis de empatia de estudantes de medicina. Revista Brasileira de Educação Médica, 2018; 42 (1): 152-160.

6. NORCINI JJ, et al. The Mini-CEX: A method for Assessing Clinical Skills. Ann Intern Med, $2003 ; 138$ (1): $476-481$.

7. SCHEFFER, M. et al. Demografia Médica no Brasil 2018. Faculdade de Medicina da Universidade de São Paulo, 2018; 20(3): 1-286.

8. SHAPIRO J. Walking a mile in their patients' shoes: empathy and othering in medical students' education. Philosophy, Ethics, and Humanities in Medicine, 2008; 3 (10): 1-11.

9. SILVA JMS, MARIN AJ. Avaliação do desempenho individual: Um estudo do uso da ferramenta feedback $360^{\circ}$. Dissertação (Mestrado Profissional em Saúde) - Curso de Medicina. Centro Universitário de Araraquara, Araraquara, 2016; $92 \mathrm{p}$.

10. SOUSA JCV. Implementação da avaliação $360^{\circ}$ do residente no programa de residência médica de cardiologia e clínica médica do hospital universitário Onofre Lopes (HUOL). Dissertação (Mestrado Profissional em Ensino na Saúde) - Curso de Medicina. Universidade Federal do Rio Grande do Norte, Natal, 2015; 52 p.

11. SOUZA SV, FERREIRA BJ. Preceptoria: desafios na residência em saúde. ABCS Health Sci, 2019; 44(1): 15-21.

12. TORRES AR, et al. Ensinando a anamnese psiquiátrica para estudantes de medicina através da inversão de papéis: relato de experiência. Revista Brasileira de Educação Médica, 2019; 43 (2): 200-207. 